\title{
Relationship Between Metabolic Disorders and Breast Cancer Incidence and Outcomes. Is There a Preventive and Therapeutic Role for Berberine?
}

\author{
MASSIMILIANO CAZZANIGA and BERNARDO BONANNI
}

European Institute of Oncology, Milan, Italy

\begin{abstract}
The association between obesity/overweight and carcinogenesis is a recognized highly complex and still partially unknown process. Nevertheless, these conditions are frequently related with several pathological states such as chronic inflammation, presence of dyslipidemia and insulinresistance (metabolic disorders) which are now accepted features contributing to the increased hormonal-dependent cancer risk. Breast cancer incidence and outcome is strictly related to metabolic disorders. Thus, managing these emerging risk factors, should be a new and optimal strategy in breast cancer prevention and therapy. Unfortunately, the agents able to interfere with metabolic disorders, produce often light or serious side-effects and consequently their compliance and efficacy are weak. Some nutraceutical compounds seem to be an ideal option with the same activity and effectiveness to ordinary agents but with minor side effects. Berberine, an extraordinary medicinal herb, has been proven to have many clinical pharmacological effects, including lowering of blood glucose, increasing insulin sensitivity, and correcting lipid metabolism disorders. It has a comparable therapeutic effect to common drugs. It acts contemporarily on hyperlipidemia, hyperglycemia and insulin resistance without their related side effects and could be a real alternative in healthy high risk or affected breast cancer patients with metabolic disorders. This commentary examines the pathophysiology of metabolic disorders and its relationship to breast cancer. Moreover, it evaluates the possible role of berberine in the clinical practice.
\end{abstract}

Correspondence to: Massimiliano Cazzaniga, European Institute of Oncology, Via Ripamonti 435, 20141, Milan, Italy. Tel: +39 0294372656, Fax: +39 0294379225, e-mail: massimiliano. cazzaniga@ieo.it

Key Words: Obesity, dyslipidemia, berberine, insulin-resistance, breast cancer, nutraceuticals, review.
Cancer is a genetic disease caused by damage in the genome of the cells. Every mutation which occurs at the genomic level is reflected on several pathways generally related to cellular activity. This damage is often acquired throughout life and directly related to a wrong lifestyle (1). The major unhealthy behaviors in developed countries are the tobacco use and dietary/lifestyle factors which taken together they represent more than two-thirds of the acquired risk factors of cancer development. In particular obesity and overweight have become major public health concerns during the last few decades as they are key risk factors for several diseases such as type-2 diabetes, cardiovascular conditions, dyslipidemia, hypertension and some cancers (2). The precise mechanisms that link obesity and cancers remain unclear - particularly in relation to the sequence of events. However, some metabolic changes in the surrounding microenvironment as chronic inflammation state, presence of dyslipidemia and insulin-resistance (generally defined as metabolic disorders) are now substantially accepted features (3) which contribute to increased cancer risk on hormonaldependent neoplasms, breast cancer included, and a potential target for conventional and nutraceutical therapies.

\section{Metabolic Disorders and Breast Cancer Risk}

The association of obesity with cancer and the ongoing obesity epidemic evolution have stimulated several researches in this particular setting. Over the past 40 years the prevalence of obesity and overweight condition has increased epidemically worldwide particularly in developed countries (4). These conditions are recognized risk factors for several diseases but recently a great deal of evidence has suggested that they negatively affect cancer risk and outcome. Growing evidence from epidemiological, preclinical and clinical studies indicates that increased adiposity is associated with increases in cancer incidence and mortality (5). A relative recent meta-analysis and systematic review shows that an 
increase in the body mass index (BMI) is associated with an enhanced risk of several malignancies and overall with $14 \%$ of all cancer deaths in men and with $20 \%$ of all cancer deaths in women (6). Recently, the population-attributable fraction (PAF), the proportion of cancers potentially avoidable if the obesity-cancer association is causal, for obesity and cancer risk worldwide has been calculated (7). Approximately $3.6 \%$ of all new cancers may be due to high BMI. Interestingly, the PAF in women is more than double than that in men, reflecting the strong relationship between obesity and hormonal cancers such as breast cancer as well. Unfortunately, cancer risk is not only related to obesity or overweight conditions but is also high in presence of metabolic syndrome (MS). MS is characterized by a cluster of endocrine disturbances including mild obesity (particularly central adiposity), glycemic perturbations (particularly the presence of insulin-resistance), raised blood pressure, elevated triglyceride levels and low high-density lipoprotein (HDL) cholesterol levels (8).

Several studies have shown the relationship between these metabolic conditions and the incidence and outcome of mammary neoplasia. A recent case-cohort study on more than 22,000 subjects showed that the presence of metabolic syndrome was associated with significantly increased breast cancer risk in all and in particular in postmenopausal women $(\mathrm{HR}=1.52,95 \% \mathrm{CI}=1.14-2.02)$ and authors suggested that a preventive approach to metabolic syndrome through lifestyle changes could confer protection against breast cancer incidence and prognosis (9). More recently, in a prospective analysis of a cohort of almost 21,000 postmenopausal women, authors found that obese and metabolically unhealthy subjects were associated with the highest risk $(\mathrm{HR}=1.62 ; 95 \% \mathrm{CI}=1.33-1.96)$ of developing cancer and suggested that both obesity and metabolic dysregulation were associated with breast cancer risk. Moreover, beyond BMI, metabolic health should be considered a clinically relevant and modifiable risk factor for breast cancer (10). A complete review of recent literature on these settings identified 11 studies that were published since 2010 (11). It was confirmed that obesity was a risk factor for breast cancer (BC) recurrence and reduced survival. It was also shown that a minor efficacy of treatment with aromatase inhibitors in hormone-receptor positive BC survivors who were obese, in comparison to normal-weight women. In addition, these analyses showed that obesity-induced changes in glucose metabolism, type-2 diabetes, and metabolic syndrome contributed to negative outcomes in BC survivors.

Along with the increasing prevalence of obesity and overweight worldwide, there is an increasing prevalence of type 2 diabetes. Potential interactions between diabetes and breast cancer are complex. Recent research has focused on the effect of this comorbid condition on incidence and mortality in women with breast cancer. The Nurses' Health
Study has reported a $17 \%$ increased risk of breast cancer incidence ( $\mathrm{HR}=1.17$ 95\% CI=1.01-1.35) in women with diabetes compared with women without this condition. This relationship was predominant in postmenopausal subjects and seemed to be related to estrogen receptor (ER)-positive cancer (12). Not only incidence, but also breast cancer outcome seemed to be related with diabetic and pre-diabetic conditions. A recent authoritative systematic review and meta-analysis examining the effect of pre-existing diabetes on breast cancer-related outcomes has shown that patients with breast cancer and diabetes had a significantly higher allcause mortality risk $(\mathrm{HR}=1.49 ; 95 \% \mathrm{CI}=1.35$ to 1.65$)$ compared with their nondiabetic counterparts (13). Authors found also that a pre-existing diabetes condition is associated with a more advanced stage at presentation. Some clinical studies have also examined the association between glycemic control and cancer risk and in a meta-analysis of 14 studies, authors reported an increased risk of breast cancer associated with a HbA1c above $8.5 \%$. (14). Compared with their nondiabetic counterparts, patients with breast cancer and preexisting diabetes and in general with metabolic disorders involving dislypidemic conditions too, have a greater risk of cancer incidence and death and tend to present at later stages of disease. Thus, we investigated the pathophysiologic interactions between metabolic disorders and breast cancer and determine whether improvements in their care can be beneficial.

\section{The Links Between Metabolic Disorders and Breast Cancer}

The relationship between metabolic disorders and breast carcinogenesis is complex and not fully understood. Several pathways related to metabolic abnormalities concur to cancer development and progression, but the importance of each one and the correct sequence of events remains substantially unclear. Fat is recognized as a "real organ" with both metabolic and endocrine functions. Multiple factors like chronic inflammation, insulin-resistance, dyslipidemia and endocrine products associated with abnormal excess and accumulation of fat are certainly related with cancer progression.

There is growing evidence that inflammation is a central and reversible mechanism through which obesity promotes cancer risk and progression. The precise physiological events leading to the initiation of an inflammatory response in obesity remains incompletely understood, but a large number of macrophages that comprise up to $40 \%$ of resident cells in adipose tissue, remain the main source of proinflammatory cytokines such as tumor necrosis factor(TNF-) and interleukins (IL-6-4-10-12) (15-17). Moreover, the chemokines secreted by adipose tissue macrophages recruit additional leukocytes, thus maintaining and expanding the chronic and subclinical inflammatory process. 
Chronic inflammation also influences the production (and their ratio) of leptin and adiponectin, two proteins secreted by adipocytes which are inversely related with carcinogenesis. Adiponectin, in contrast to leptin, is in fact inversely associated with obesity and hyperinsulinemia (18) and appears to have anti-inflammatory, anti-angiogenic, proapoptotic and anti-diabetic properties too (19). Moreover, recent evidence demonstrates that the Leptin-Adiponectin $(\mathrm{L}-\mathrm{A})$ ratio could be a useful index and surrogate marker of insulin-resistance state (IR) and its associated cancer risk. In accordance with these biological activities, an increased $\mathrm{L}-\mathrm{A}$ ratio has been associated with risk for several types of cancer including breast.

Systemically, the metabolic syndrome, including dyslipidemia and insulin resistance, occurs in the setting of adipose inflammation and operates in concert with local mechanisms to sustain the inflammatory microenvironment and promote tumor growth. Importantly, adipose inflammation and its pro-tumor consequences, insulin resistance and dyslipidemia in particular can be found in some individuals who are not considered to be obese or overweight by body mass index.

\section{Insulin Resistance}

Obesity increases glycemic perturbations and the related cancer risk through induction of insulin resistance. Recently, a new energy-based concept was hypothesized in which insulin resistance is a result of energy surplus in cells and the keys of this relationship are the mitochondrial dysfunction and the reduction of activity of adenosine monophosphate-activated protein kinase (AMPK) (20). Recent evidence suggests that inhibition of the nutrientsensing enzyme AMPK is an early event in the development of IR in response to high glucose and fatty acids (FA). Practically, presence of obesity or overweight guarantees an ideal substrate to the cells, mainly composed by glucose and fatty acids. In this scenario, there is a mitochondrial overactivation with a surplus of energy production (ATP) that leads to the AMPK signalling pathways inactivation. AMPK is an energy sensor which has also an insulin-sensitizing action on resistant cells and its inactivation by energetic surplus production leads to an insulin resistance state. The complete mechanism that links obesity and IR is summarized in Figure 1.

Moreover, over this insulin-sensitizing action, AMPK has also a direct effect on carcinogenesis. According to several published studies (21), AMPK activation strongly suppresses cell proliferation in both malignant and non-malignant cells. AMPK actions seem to be mediated by multiple mechanisms: on cell cycle progression with activation of p53 suppressor gene and on protein synthesis inhibition by blocking the mTOR pathway.

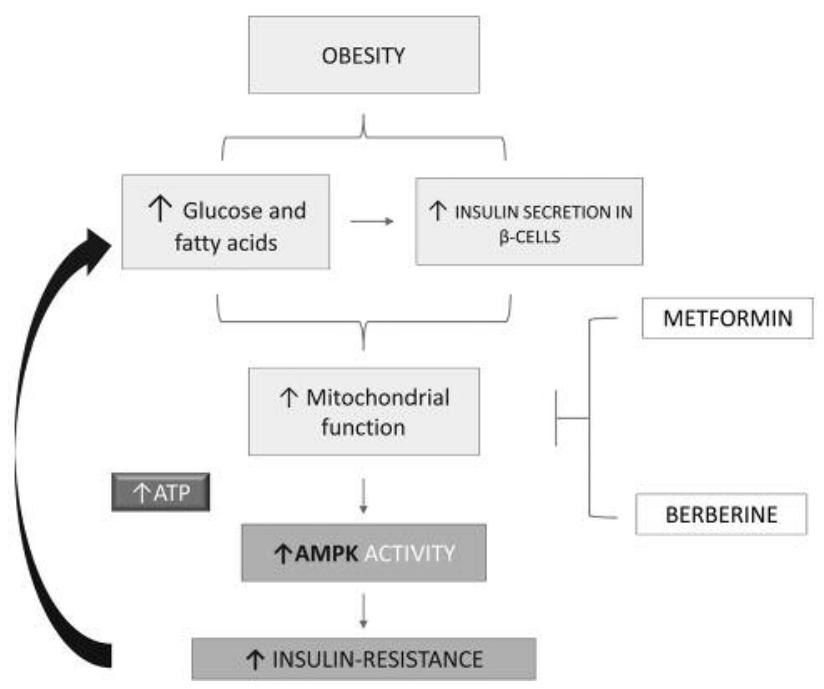

Figure 1. In obesity, AMPK activity is reduced by the mitochondrial over-activation through energetic substrates. Drugs as metformin or berberine are able to stop the mitochondrial function and restore insulin sensitivity.

Presence of IR is directly and indirectly linked to breast cancer risk and outcome. The direct way is named "insulincancer hypothesis" that postulates that the condition of IR has two metabolic consequences both related to cancer disease. The first one involves the insulin-IGF axis and is characterized by the presence of chronic hyperinsulinemia. Insulin is a mutagenic factor which is considered as an independent risk factor for breast cancer and it may have a substantial role in explaining the obesity-breast cancer relationship (22). Generally, its increase is also associated with decreased concentrations of insulin-like growth factor binding protein 1 (IGFBP-1) and IGFBP-2, leading to increased availability of IGF-I and by binding to its cellular receptor produces concomitant changes in the cellular environment that favors tumor formation. In contrast, the IR indirect effect on carcinogenesis is related to the presence of hyperglycemia and the metabolic reprogramming which occurs in cancer cells (23). Metabolic shift from catabolic to anabolic metabolism is a classic hallmark of cancer cells. This metabolic reprogramming, known as the Warburg effect (24), results in the use of aerobic glycolysis in preference to oxidative phosphorylation (OXPHOS) for increased energy production in cancer cells. This condition is closely related to glucose availability because cancer cells consume an excessive quantity of glucose, and the presence of hyperglycemia related to IR ensures an ideal microenvironment for cancer cell proliferation and growth. Several clinical studies have confirmed this relationship between IR and cancer incidence. A recent cross-sectional 


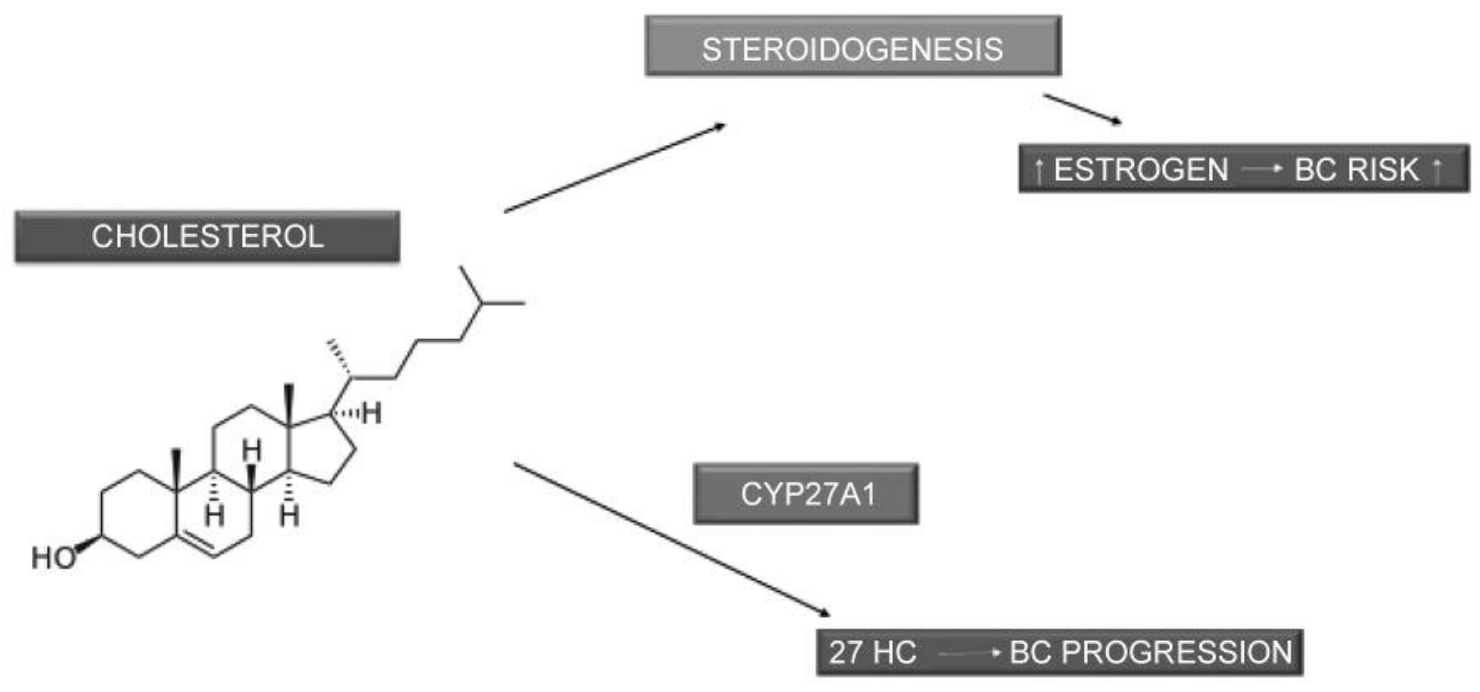

Figure 2. Direct and indirect relationship between cholesterol and hormonal effects.

study regarding female cancers, conducted in 121,230 middle-aged and elderly non-diabetic women has shown that the prevalence of both premenopausal and postmenopausal breast cancer, postmenopausal ovarian cancer and premenopausal endometrial cancer was higher in insulinresistant participants than in insulin-sensitive participants (25). Subgroup analysis revealed that the positive association between insulin resistance and risk of prevalent breast cancer was observed in a population with younger age, overweight or obese, and with impaired glucose tolerance (IGR).

Thus, suppression of mitochondrial ATP production and activation of AMPK, for example with metformin or with an alternative compound such as berberine (both recognized mitochondrial inhibitors and AMPK activators), could be a promising approach in the treatment of IR and breast cancer related to this condition.

\section{Dyslipidemia}

Presence of metabolic disorders including those involved in cholesterol biosynthesis are implicated in cancer development and progression. The accumulation of cholesterol is a general feature of cancer tissue and recent evidence suggests that hypercholesterolemia plays a critical role in the progression of several cancers, breast cancer included $(26,27)$. Cholesterol is synthesized through the mevalonate pathway, with a series of reactions originally regulated by the HMG-CoA reductase action (28). Studies on cancer cells revealed that cholesterol synthesis is enhanced in comparison with healthy tissue (29) and this suggests the importance of the mevalonate pathway in cancer metabolism and its role as a potential target for therapeutic strategies. In detail, cholesterol is an essential component of breast cell membranes as well as precursor of steroid hormones and the hypothesis that breast cancer cells need excess cholesterol and intermediates of its metabolism, to maintain a high level of proliferation, is substantially accepted.

Endocrine tissues such as the gonads and the adrenals are tissues that are able to produce active steroid hormones from cholesterol and to deliver them into the circulation (30). Steroid hormones have long been recognized as regulators of cell proliferation and differentiation and are intimately associated with the etiology of breast cancer. In addition to these potential direct effects of cholesterol, recent works have identified its primary metabolite, oxysterol, the 27hydroxycholesterol $(27 \mathrm{HC})$ synthesized by the cytochrome P450 oxidase, sterol 27-hydroxylase (CYP27A1), as a further molecule related to carcinogenesis (31). $27 \mathrm{HC}$ can bind to and modulate the activity of both $\operatorname{ER} \alpha$ and $\operatorname{ER} \beta$ and especially on the $\alpha$-subunit, it is able to increase breast cancer cellular proliferation and tumor growth (32) (Figure 2). In conclusion, high cholesterol levels can stimulate breast cancer development and progression in a double way, increasing steroid hormone production and/or create an active metabolite able to stimulate the estrogen pathway.

\section{Conventional Therapeutic Intervention}

The association between insulin-resistance, dyslipidemia and breast cancer development and progression suggests that managing these emerging conditions and their related carcinogenic pathways, should be a new and optimal strategy in breast cancer prevention and therapy. Several 
studies have also recently examined whether cholesterollowering medication (CLM) such as statins and insulinsensitizing medications (metformin) could be efficacious in this setting $(33,34)$.

Interestingly, cancer patient's outcome seems to be strictly related to statin or CLM use. Recently, the Breast International Group (BIG) conducted a randomized, phase III, double blinded trial (BIG 1-98) which enrolled more than 8,000 breast cancer postmenopausal women. The trial was designed to answer two questions concerning how best to use endocrine agents for the treatment of early breast cancer in postmenopausal women with hormone receptor-positive tumors. The first was to compare letrozole monotherapy with tamoxifen monotherapy and the second to determine the benefit of letrozole in sequence with tamoxifen (35). In addition to the letrozole superiority, authors also found an important effect related to the patient's metabolic condition. Given the data from previous studies on the mechanism of action of CLM against breast cancer, they hypothesized that CLM in combination with endocrine therapy may improve breast cancer outcome. Three time-to-event endpoints were considered as disease-free survival, breast cancer-free interval and distant recurrence-free interval (36). Interestingly the authors concluded that in 789 patients with breast cancer who used CLM at study baseline had more beneficial tumor characteristics and improved outcomes compared with non-users. The prognostic impact of CLM initiation during endocrine therapy showed beneficial effects of initiating CLM, although the benefit may be restricted to patients who received aromatase inhibitors treatment. Study results suggest that high cholesterol level can reduce the aromatase inhibitors efficacy thus increasing the recurrence rate or in other words the use of CLM during adjuvant therapy may have a role in preventing breast cancer recurrence in ER positive early stage breast cancer.

If statins are the best CLMs, metformin is a biguanide derivative, currently approved for the treatment of noninsulin dependent diabetes mellitus, and an insulinsensitizing agent with potent antihyperglycemic properties. In the last decades, several studies have shown its anticancer properties on incidence and prognosis of many types of malignancies (37). Researchers suggest that metformin may have different mechanisms of tumor inhibition both via insulin-dependent and independent pathways (38), including activation of AMPK with a consequent inhibition in cancer proliferation and induction of apoptosis (39). An important systematic review and meta-analysis was performed to assess the effect of metformin on cancer incidence and mortality in diabetic patients and suggested that the agent is associated with a decreased risk of cancer incidence (37). In a breast cancer setting another meta-analysis confirmed these data and supported the protective effect of metformin on breast cancer risk among postmenopausal women with diabetes (40). In non-diabetic subjects, a recent pre-surgical trial evaluated changes in Ki-67 between pre-treatment biopsy and post-treatment surgical specimens and indicated that Ki-67 had a prognostic value and might predict antitumor activity in breast cancer (41). The research showed that metformin administered before surgery did not significantly reduce Ki-67 overall but had a significantly opposite effect depending on IR, particularly on luminal B tumors. There was a proportional decrease in $\mathrm{Ki}-67$ of $10.5 \%$ in women with a homeostasis model assessment (HOMA-index) score $>2.8$, while an opposite increase of $11.1 \%$ was observed in women with a HOMA score $<2.8$. Interestingly, similar drug effects on $\mathrm{Ki}-67$ were noted to be related to $\mathrm{BMI}$, waist/hip ratio, alcohol consumption and C-reactive protein (CRP). Moreover, an overall reduction in CRP and total cholesterol were noted in the metformin group. These findings confirm that metabolic disorders directly influence cancer risk and outcome, and so these disorders should be treated to combat cancer.

Unfortunately, the agents that have already demonstrated their ability to interfere with metabolic disorders, are limited in their clinical use. They can produce light or serious side effects with consequent weak compliance and efficacy (Figure 3). Moreover, patients often refuse a statin or metformin treatment for a long time period because they perceive it as an overtreatment especially in case of an ongoing adjuvant treatment (i.e. aromatase inhibitors). For these reasons, we urgently need to find alternative/synergistic compounds with similar activity and effectiveness but with fewer or no side effects. Recently, several researchers have directed their attention on nutraceutical compounds, which seem to be safe and effective to modulate metabolic disorders and give us an authoritative clinical alternative.

\section{Berberine}

Berberine (BBR) is a quaternary ammonium salt from the protoberberine group, highly concentrated in the roots, rhizomes and stem bark of various plants as Berberis gender. BBR has been used since a very long time in clinical practice as a luminally acting agent with different anti-diarrheal mechanisms including bactericidal activity (42). As reported below, a great number of reports demonstrated the effectiveness of BBR supplementation in lipid disorders and hyperglycemia. The major disadvantage of BBR is its poor oral bioavailability which is attributed mainly to a P-glycoprotein (P-gp)-mediated gut extrusion process (43). This limit has been overcome with its combination with a potent P-gp inhibitor such as silymarin (from Silybum marianum) (44). A combination of BBR and silymarin has shown greater biodisponsibility and consequently good clinical effectiveness in reducing cholesterol and glycaemia 


\begin{tabular}{|c|c|}
\hline Statin side effect & Metformin side effect \\
\hline $\begin{array}{l}\text { Common side effect of all statins } \\
\text { - Muscle pain is the most common side effect caused by } \\
\text { statin use. } \\
\text { Symptoms could be: } \\
\text { - Unusual muscle pain or cramps } \\
\text { - Tiredness } \\
\text { - } \text { - Dever } \\
\text { - Diarrhoea }\end{array}$ & $\begin{array}{l}\text { Very common } \\
\text { - Nausea } \\
\text { - Vomiting } \\
\text { - Aiarrhoea } \\
\text { Very rare } \\
\text { - Elevated levels of lactic acid in the blood (lactic } \\
\text { acidosis) } \\
\text { - Decreased absorption of vitamin B12 during } \\
\text { long-term use }\end{array}$ \\
\hline $\begin{array}{l}\text { These could be symptoms of rhabdomyolysis, a dangerous } \\
\text { muscle condition that can cause kidney problems }\end{array}$ & - Skin reactions such as rash, itching or flushing \\
\hline
\end{tabular}

Figure 3. Metformin and statins' side effects.

than BBR extract alone (45). BBR has been shown to regulate both glucose and lipid metabolism in vitro and in vivo, and so could be administered alone or together with other nutraceuticals or conventional drugs to manage metabolic disorders associated with increased cancer risk and progression.

Several mechanisms of action are involved in its dual ability on modulating a lipid and glycemic profile. Adequate evidences supports the potential role of BBR as an antidiabetic agent mainly due to its action on AMPK signaling pathway (activator) with subsequent reduction of insulin-resistance state and induction of glycolysis (46). In detail, in the glycemic setting, BBR has demonstrated its effectiveness in diabetic patients, significantly decreasing fasting and postprandial blood glucose and glycosylated haemoglobin (HbA1c) levels $(47,48)$. BBR regulates glucose metabolism by stimulating glucose uptake by glucose transporter type 4 (GLUT-4) up-regulation (49) and activating 5' adenosine monophosphate-activated protein kinase ( $5^{\prime}$ AMPK) because of inhibition of mitochondrial function (50). Interestingly, these different mechanisms mean BBR can be combined with other glucose-lowering agents (51) in order to increase efficacy without increasing side effects.

In addition, BBR acts on lipid profile principally increasing expression of the hepatic LDL receptor (LDLR). BBR can increase the available receptors by the inhibition of Pro-protein-Convertase-subtilisin-Kexin-9 (PCSK9) (52). These mechanisms are distinct from those recognized by statins therapy, which instead decrease cholesterol biosynthesis through HMG-CoA reductase inhibition (53). Moreover, the cholesterol-lowering action of BBR could be increased by adding monacolins, which are naturally derived statins and are relatively safe compounds derived from Monascus purpureus/Red Yeast Rice (RYR) fermentation (54). Recently, RYR has been formulated together with berberine from $B$. aristata (55). This association is thought to generate a pharmacodynamic synergy due to the opposing effects exerted by berberine and monacolins on PCSK 9 and HMG-CoA reductase (Figure 4).

Several randomized studies have confirmed the efficacy of $B B R$ on glycemic and lipidemic pathways modulation. A systemic review and meta-analysis of RCTs showed that BBR was effective in treating hyperglycemia, demonstrating significant reductions in several glycemic parameters such as fasting plasma glucose levels (FPG), postprandial plasma glucose levels (PPG) and HbA1c (48). Moreover, compared to oral hypoglycemic drugs alone, BBR administered with the same oral hypoglycemics showed a better glycemic control. No serious adverse effects from BBR were reported (51). Another meta-analysis of about twenty-seven randomized controlled clinical trials including more than 2,500 patients indicated that BBR has comparable therapeutic effects on type 2DM, hyperlipidemia and hypertension with no serious side effects (56). Considering the relatively low cost compared to other first-line medicines and treatments, BBR might be a good alternative for low socioeconomic status patients to treat type 2DM, hyperlipidemia, and hypertension over a long time period.

In the lipid profile, data are equally consistent. A recent systemic review and meta-analysis of RCTs showed that administration of BBR produced a significant reduction in the levels of total cholesterol $(-25 \%)$, triglycerides $(-20 \%)$ and low-density lipoprotein cholesterol (-30\%), with a 


\section{BERBERINE ON GLICEMIC PROFILE}

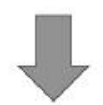

BERBERINE ON LIPIDIC PROFILE

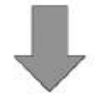

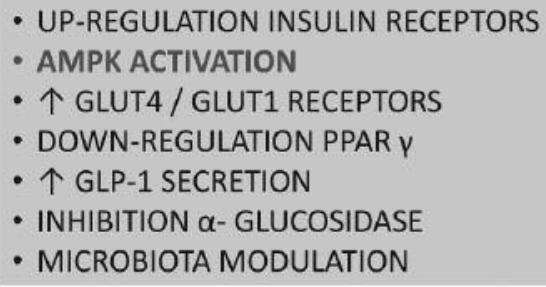

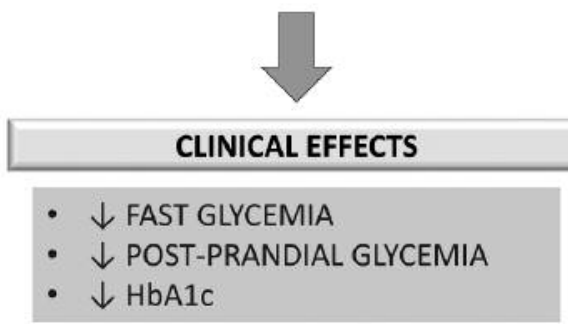

- UP-REGULATION LDL RECEPTOR

- DOWN-REGULATION OF PCSK-9

- PPAR- $\alpha$ MODULATION

- $\downarrow$ CHOLESTEROL INTESTINAL ABSORPTION

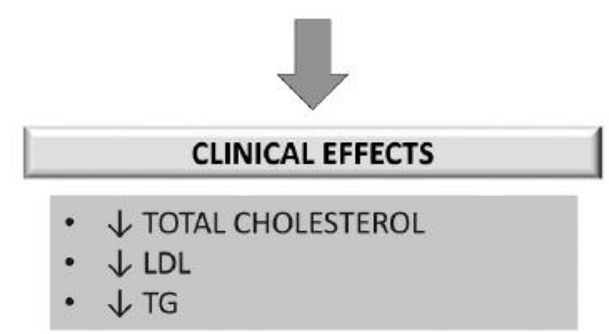

Figure 4. Berberine's mechanisms of action.

remarkable increase in HDL (47). In monotherapy, BBR in more than one hundred euglycemic, overweight dyslipidemic patients showed to be a safe and effective compound in improving the lipid profile, insulin-resistance state and adipocytokines levels (57). In addition, in 136 obese and T2DM subjects it showed the ability to ameliorate lipid and glycemic profiles, visceral adiposity and blood pressure parameters (58). The different mechanisms of action compared to statins, lead to the possibility to increase the efficacy of BBR which can be used as an add-on therapy condition too. Kong et al. in 2008 (59) suggested that a combination therapy for hyperlipidemia using BBR and simvastatin, could be a new regimen for hypercholesterolemia. Furthermore, add-on therapy regimen seems to be a good alternative in order to control statins side effects. A recent study (60) suggests the possibility to give half of the previously taken dose of statins, in patients intolerant to conventional doses, in addition to BBR, without decreasing the efficacy. Finally, research has been done to evaluate effectiveness of BBR plus monacolins, formulation. The effects of a cholesterollowering supplement containing berberine, silymarin, and MK-20 (BSM) in patients with dyslipidemia were clinically analyzed in a recent authoritative Italian work (61). In particular, the clinical role of BSM in naïve and in statinintolerant patients was retrospectively evaluated and the effects observed were compared with those obtained in patients without treatment or treated with lovastatin. Total cholesterol, low density lipoprotein, and triglyceride levels were lower, and the creatine phosphokinase increase was reduced in patients treated with BSM compared to those treated with lovastatin. Similar results were also obtained in statin-intolerant subjects where BSM was administered as add-on therapy to other compounds such as ezetimibe or fenofibrate. Authors concluded that BSM was a food supplement potentially useful 1) as a primary intervention in subjects with dyslipidemia 2) as add-on therapy in mildly statin-intolerant patients; and 3) in dyslipidemic patients with a negative perception of statins who preferred a treatment seen as natural.

\section{Conclusion}

Metabolic disorders define a series of alterations involved in both lipid and glycemic profiles and by now they are recognized risk factors for the incidence and outcome of several kinds of cancer in particular -but not only- those that are hormone-dependent, such as breast cancer. The mechanisms which link metabolic disorders and cancer are complex and not fully understood, but certainly driven by the presence of insulin-resistance, dyslipidemia and inflammatory state. The agents able to interfere with these pathways have both light and serious side-effects. For these reasons, we need to find alternative and/or synergistic agents safer than these. A natural compound like BBR, has abundantly shown in mono and in add-on therapy profiles, its ability to modulate these pathways without limiting side-effects and could be a 
safe and valid alternative to conventional drugs in breast cancer prevention and therapy.

The authoritative and abundant data present in the literature have pushed important Italian and European societies on cardiovascular and diabetic diseases, to insert BBR in their joint position statement and guidelines in order to accept and advise the use of BBR in selected patients (62, 63). We suggest it is time to consider this approach in cancer and in a breast cancer setting too.

\section{References}

1 Othman NH: Honey and cancer: sustainable inverse relationship particularly for developing nations-a review. Evid Based Complement Alternat Med 2012: 410406, 2012.

2 Must A, Spadano J, Coakley EH, Field AE, Colditz G and Dietz WH: The disease burden associated with overweight and obesity. JAMA 282: 1523-1529, 1999.

3 Iyengar NM, Gucalp A, Dannenberg AJ and Hudis CA: Obesity and cancer mechanisms: tumor microenvironment and inflammation. J Clin Oncol 34: 4270-4276, 2016.

4 Finucane MM, Stevens GA, Cowan MJ, Danaei G, Lin JK, Paciorek CJ, Singh GM, Gutierrez HR, Lu Y, Bahalim AN, Farzadfar F, Riley LM and Ezzati M: National, regional, and global trends in body-mass index since 1980: systematic analysis of health examination surveys and epidemiological studies with 960 country-years and 9.1 million participants. Lancet 377: 557567, 2011.

5 Keum N, Greenwood DC, Lee DH, Kim R, Aune D, Ju W, Hu FB and Giovannucci EL: Adult weight gain and adiposity-related cancers: a dose-response meta-analysis of prospective observational studies. J Natl Cancer Inst 107, 2015. doi: 10.1093/jnci/djv088

6 Renehan AG, Tyson M, Egger M, Heller RF and Zwahlen M: Body-mass index and incidence of cancer: a systematic review and meta-analysis of prospective observational studies. Lancet 371: 569-578, 2008.

7 Arnold M, Pandeya N, Byrnes G, Renehan PAG, Stevens GA, Ezzati PM, Ferlay J, Miranda JJ, Romieu I, Dikshit R, Forman D and Soerjomataram I: Global burden of cancer attributable to high body-mass index in 2012: A population-based study. Lancet Oncol 16: 36-46, 2015.

8 Esposito K, Chiodini P, Colao A, Lenzi A and Giugliano D: Metabolic syndrome and risk of cancer: a systematic review and meta-analysis. Diabetes Care 35: 2402-2411, 2012.

9 Agnoli C, Grioni S, Sieri S, Sacerdote C, Ricceri F, Tumino R, Frasca G, Pala V, Mattiello A, Chiodini P, Iacoviello L, De Curtis A, Panico S and Krogh V: Metabolic syndrome and breast cancer risk: a case-cohort study nested in a multicentre italian cohort. PLoS One 10: e0128891, 2015.

10 Kabat GC, Kim MY, Lee JS, Ho GY, Going SB, BeebeDimmer J, Manson JE, Chlebowski RT and Rohan TE: Metabolic obesity phenotypes and risk of breast cancer in postmenopausal women. Cancer Epidemiol Biomarkers Prev 26: 1730-1735, 2017.

11 Azrad M and Demark-Wahnefried W: The association between adiposity and breast cancer recurrence and survival: A review of the recent literature. Curr Nutr Rep 3: 9-15, 2014.
12 Michels KB, Solomon CG, Hu FB, Rosner BA, Hankinson SE, Colditz GA, Manson JE and Nurses' Health Study: Type 2 diabetes and subsequent incidence of breast cancer in the Nurses' Health Study. Diabetes Care 26: 1752-1758, 2003.

13 Peairs KS, Barone BB, Snyder CF, Yeh HC, Stein KB, Derr RL, Brancati FL and Wolff AC: Diabetes mellitus and breast cancer outcomes: a systematic review and meta-analysis. J Clin Oncol 29: 40-46, 2011.

14 De Beer JC and Liebenberg L: Does cancer risk increase with HbA1c, independent of diabetes? Br J Cancer 110: 2361-2368, 2014.

15 Weisberg SP, McCann D, Desai M, Rosenbaum M, Leibel RL and Ferrante AW: Obesity is associated with macrophage accumulation in adipose tissue. J Clin Investig 112: 1796-1808, 2003.

16 Barbarroja N, Lopez-Pedera R, Mayas MD, García-Fuentes E, Garrido-Sánchez L, Macías-González M, El Bekay R, VidalPuig A and Tinahones FJ: The obese healthy paradox: Is inflammation the answer? Biochem J 430: 141-149, 2010.

17 Barbarroja N, Lopez-Pedrera C, Garrido-Sanchez L, Mayas MD, Oliva-Olivera W, Bernal-Lopez MR, El Bekay R and Tinahones FJ: Progression from high insulin resistance to type 2 diabetes does not entail additional visceral adipose tissue inflammation. PLoS One 7: e48155, 2012.

18 Mohammadzadeh G and Zarghami N: Hypoadiponectinemia in obese subjects with type II diabetes: a close association with central obesity indices. J Res Med Sci 16: 713-723, 2011.

19 Roberts DL, Dive C and Renehan AG: Biological mechanisms linking obesity and cancer risk: new perspectives. Annu Rev Med 61: 301-316, 2010.

20 Zhang Y and Ye J: Mitochondrial inhibitor as a new class of insulin sensitizer. Acta Pharm Sin B 2: 341-349, 2012.

21 Ikhlas S and Ahmad M: Metformin: Insights into its anticancer potential with special reference to AMPK dependent and independent pathways. Life Sci 185: 53-62, 2017.

22 Gunter MJ, Hoover DR, Yu H, Wassertheil-Smoller S, Rohan TE, Manson JE, Li J, Ho GY, Xue X, Anderson GL, Kaplan RC, Harris TG, Howard BV, Wylie-Rosett J, Burk R and Strickler HD: Insulin, insulin-like growth factor-I, and risk of breast cancer in postmenopausal women. J Natl Cancer Inst 101: 48$60,2009$.

23 Cazzaniga M and Bonanni B: Relationship between metabolic reprogramming and mitochondrial activity in cancer cells. Understanding the anticancer effect of metformin and its clinical implications. Anticancer Res 35: 5789-5796, 2015.

24 Warburg O: On the origin of cancer cells. Science 123: 309-314, 1956.

25 Sun W, Lu J, Wu S, Bi Y, Mu Y, Zhao J, Liu C, Chen L, Shi L, Li Q, Yang T, Yan L, Wan Q, Liu Y, Wang G, Luo Z, Tang X, Chen G, Huo Y, Gao Z, Su Q, Ye Z, Wang Y, Qin G, Deng H, Yu X, Shen F, Chen L, Zhao L, Wang T, Sun J, Xu M, Xu Y, Chen Y, Dai M, Zhang J, Zhang D, Lai S, Li D, Ning G and Wang W: Association of insulin resistance with breast, ovarian, endometrial and cervical cancers in non-diabetic women. Am J Cancer Res 6: 2334-2344, 2016.

$26 \mathrm{Kuzu}$ OF, Noory MA and Robertson GP: The role of cholesterol in cancer. Cancer Res 76: 2063-2070, 2016.

27 Nelson ER, Chang CY and McDonnell DP: Cholesterol and breast cancer pathophysiology. Trends Endocrinol Metab 25: 649-655, 2014 
28 Singh P, Saxena R, Srinivas G, Pande G and Chattopadhyay A: Cholesterol biosynthesis and homeostasis in regulation of the cell cycle. PLoS One 8: e58833, 2013.

29 Clendening JW, Pandyra A, Boutros PC, El Ghamrasni S, Khosravi F, Trentin GA, Martirosyan A, Hakem A, Hakem R, Jurisica I and Penn LZ: Dysregulation of the mevalonate pathway promotes transformation. Proc Natl Acad Sci USA 107: 15051-15056, 2010.

30 Miller WL and Auchus RJ: The molecular biology, biochemistry, and physiology of human steroidogenesis and its disorders. Endocr Rev 32: 81-151, 2011.

31 Nelson ER, Wardell SE, Jasper JS, Park S, Suchindran S, Howe MK, Carver NJ, Pillai RV, Sullivan PM, Sondhi V, Umetani M, Geradts J and McDonnell DP: 27-Hydroxycholesterol links hypercholesterolemia and breast cancer pathophysiology. Science 342: 1094-1098, 2013.

32 DuSell CD and McDonnell DP: 27-Hydroxycholesterol: a potential endogenous regulator of estrogen receptor signaling. Trends Pharmacol Sci 29: 510-514, 2008.

33 Kuoppala J, Lamminpää A and Pukkala E: Statins and cancer: A systematic review and meta-analysis. Eur J Cancer 44: 21222132, 2008.

34 Goodwin PJ, Ligibel JA and Stambolic V: Metformin in breast cancer: time for action. J Clin Oncol 27: 3271-3273, 2009.

35 Breast International Group (BIG) 1-98 Collaborative Group: A comparison of letrozole and tamoxifen in postmenopausal women with early breast cancer. N Engl J Med 354: 2200 2006.

36 Borgquist S, Giobbie-Hurder A, Ahern TP, Garber JE, Colleoni M, Láng I, Debled M, Ejlertsen B, von Moos R, Smith I, Coates AS, Goldhirsch A, Rabaglio M, Price KN, Gelber RD, Regan $\mathrm{MM}$ and Thürlimann B: Cholesterol, cholesterol-lowering medication use, and breast cancer outcome in the BIG 1-98 study. J Clin Oncol 35: 1179-1188, 2017.

37 Decensi A, Puntoni M, Goodwin P, Cazzaniga M, Gennari A, Bonanni B and Gandini S: Metformin and cancer risk in diabetic patients: a systematic review and meta-analysis. Cancer Prev Res (Phila) 3: 1451-1461, 2010.

38 Gonzalez-Angulo AM and Meric-Bernstam F: Metformin: a therapeutic opportunity in breast cancer. Clin Cancer Res 16: 1695-1700, 2010

39 Cazzaniga M, Bonanni B, Guerrieri-Gonzaga A and Decensi A: Is it time to test metformin in breast cancer clinical trials? Cancer Epidemiol Biomarkers Prev 18: 701-705, 2009.

40 Col NF, Ochs L, Springmann V, Aragaki AK and Chlebowski RT: Metformin and breast cancer risk: a meta-analysis and critical literature review. Breast Cancer Res Treat 135: 639-646, 2012.

41 Bonanni B, Puntoni M, Cazzaniga M, Pruneri G, Serrano D, Guerrieri-Gonzaga A, Gennari A, Trabacca MS, Galimberti V, Veronesi P, Johansson H, Aristarco V, Bassi F, Luini A, Lazzeroni M, Varricchio C, Viale G, Bruzzi P and Decensi A: Dual effect of metformin on breast cancer proliferation in a randomized presurgical trial. J Clin Oncol 30: 2593-2600, 2012

42 Imanshahidi $M$ and Hosseinzadeh H: Pharmacological and therapeutic effects of Berberis vulgaris and its active constituent, berberine. Phytother Res 22: 999-1012, 2008.

43 Pan GY, Wang GJ, Liu XD, Fawcett JP and Xie YY: The involvement of $\mathrm{P}$-glycoprotein in berberine absorption Pharmacol Toxicol 91: 193-197, 2002.
44 Zhou S, Lim LY and Chowbay B: Herbal modulation of Pglycoprotein. Drug Metab Rev 36: 57-104, 2004.

45 Di Pierro F, Putignano P, Villanova N, Montesi L, Moscatiello $\mathrm{S}$ and Marchesini G: Preliminary study about the possible glycemic clinical advantage in using a fixed combination of Berberis aristata and Silybum marianum standardized extracts versus only Berberis aristata in patients with type 2 diabetes. Clin Pharmacol 5: 167-174, 2013.

46 Pang B, Zhao LH, Zhou Q, Zhao TY, Wang H, Gu CJ and Tong XL: Application of berberine on treating type 2 diabetes mellitus. Int J Endocrinol 2015: 905749, 2015.

47 Dong H, Zhao Y, Zhao L and Lu F: The effects of berberine on blood lipids: a systemic review and meta-analysis of randomized controlled trials. Planta Med 79: 437-446, 2013.

48 Dong H, Wang N, Zhao L and Lu F: Berberine in the treatment of type 2 diabetes mellitus: a systemic review and meta-analysis. Evid Based Complement Alternat Med 2012: 591654, 2012.

49 Liu LZ, Cheung SC, Lan LL, Ho SK, Xu HX, Chan JC and Tong PC: Berberine modulates insulin signaling transduction in insulin-resistant cells. Mol Cell Endocrinol 317: 148-153, 2010.

$50 \mathrm{Zhu} \mathrm{X}$, Bian $\mathrm{H}$ and Gao X: The potential mechanisms of berberine in the treatment of nonalcoholic fatty liver disease. Molecules 21: pii: E1336, 2016.

51 Di Pierro F, Villanova N, Agostini F, Marzocchi R, Soverini V and Marchesini G: Pilot study on the additive effects of berberine and oral type 2 diabetes agents for patients with suboptimal glycemic control. Diabetes Metab Syndr Obes 5: 213-217, 2012.

52 Momtazi AA, Banach M, Pirro M, Katsiki N and Sahebkar A: Regulation of PCSK9 by nutraceuticals. Pharmacol Res 120: 157-169, 2017

53 Thurnher M, Nussbaumer O and Gruenbacher G: Novel aspects of mevalonate pathway inhibitors as antitumor agents. Clin Cancer Res 18: 3524-3231, 2012.

54 McCarty MF, O'Keefe JH and DiNicolantonio JJ: Red Yeast Rice Plus Berberine: Practical strategy for promoting vascular and metabolic health. Altern Ther Health Med 212: 40-45, 2015.

55 Millán J, Cicero AF, Torres $\mathrm{F}$ and Anguera A: Effects of a nutraceutical combination containing berberine (BRB), policosanol, and red yeast rice (RYR), on lipid profile in hypercholesterolemic patients: A meta-analysis of randomised controlled trials. Clin Investig Arterioscler 28: 178-187, 2016.

56 Lan J, Zhao Y, Dong F, Yan Z, Zheng W, Fan J and Sun G: Meta-analysis of the effect and safety of berberine in the treatment of type 2 diabetes mellitus, hyperlipemia and hypertension. J Ethnopharmacol 161: 69-81, 2015.

57 Derosa G, Bonaventura A, Bianchi L, Romano D, D' Angelo A, Fogari E and Maffioli P: Effects of Berberis aristata/Silybum marianum association on metabolic parameters and adipocytokines in overweight dyslipidemic patients. J Biol Regul Homeost Agents 27: 717-728, 2013.

58 Guarino G, Strollo F, Carbone L, Della Corte T, Letizia M, Marino $G$ and Gentile S: Bioimpedance analysis, metabolic effects and safety of the association Berberis aristata/Bilybum marianum: a 52-week double-blind, placebo-controlled study in obese patients with type 2 diabetes. J Biol Regul Homeost Agents 31: 495-502, 2017.

59 Kong WJ, Wei J, Zuo ZY, Wang YM, Song DQ, You XF, Zhao LX, Pan HN and Jiang JD: Combination of simvastatin with berberine improves the lipid-lowering efficacy. Metabolism 57: 1029-1037, 2008. 
60 Derosa G, Romano D, D'Angelo A and Maffioli P: Berberis aristata combined with Silybum marianum on lipid profile in patients not tolerating statins at high doses. Atherosclerosis 239: 87-92, 2015.

61 Di Pierro F, Putignano P, Ferrara T, Raiola C, Rapacioli G and Villanova N: Retrospective analysis of the effects of a highly standardized mixture of Berberis aristata, Silybum marianum, and monacolins $\mathrm{K}$ and $\mathrm{KA}$ in patients with dyslipidemia. Clin Pharmacol 9: 1-7, 2016.

62 Pirro M, Vetrani C, Bianchi C, Mannarino MR, Bernini F and Rivellese AA: Joint position statement on "Nutraceuticals for the treatment of hypercholesterolemia" of the Italian Society of Diabetology (SID) and of the Italian Society for the Study of Arteriosclerosis (SISA). Nutr Metab Cardiovasc Dis 27: 2-17, 2017.
63 Authors/Task Force Members:, Catapano AL, Graham I, De Backer G, Wiklund O, Chapman MJ, Drexel H, Hoes AW, Jennings CS, Landmesser U, Pedersen TR, Reiner Ž, Riccardi G, Taskinen MR, Tokgozoglu L, Verschuren WM, Vlachopoulos C, Wood DA and Zamorano JL: 2016 ESC/EAS Guidelines for the Management of Dyslipidaemias: The Task Force for the Management of Dyslipidaemias of the European Society of Cardiology (ESC) and European Atherosclerosis Society (EAS) Developed with the special contribution of the European Association for Cardiovascular Prevention \& Rehabilitation (EACPR). Atherosclerosis 253: 281-344, 2016.

Received June 5, 2018

Revised June 18, 2018

Accepted June 19, 2018 